\title{
Cuantificación de cafeína y su efecto en la calidad sensorial en tres variedades de café (Coffea arabica L.), según nivel altitudinal en la Región San Martín
}

Quantification of caffeine and its effect on sensory quality in three varieties of coffee (Coffea arabica L.), according to altitudinal level in the San Martín Region

Navarro-Ramírez, Enrique1[0000-0002-9595-8830]; Pezo-Gonzáles, Mario ${ }^{1[0000-0002-9656-1907]}$ y García-Paredes, María ${ }^{10000-0002-8334-6992]}$ ${ }^{1}$ Universidad Nacional de San Martín, Tarapoto, Perú enavarro@unsm.edu.pe

Resumen. La calidad en taza del café depende de los atributos sensoriales del grano tostado molido, cantidad de cafeína y zona agrícola de procedencia. Evaluamos el efecto de los niveles altitudinales sobre el contenido de cafeína en café tostado molido entre variedades Catimor, Caturra y Pache en la región San Martín y la relación del contenido de cafeína sobre la calidad sensorial del café en taza. Se recolectaron muestras de café en tres niveles altitudinales. Para el análisis sensorial se aplicó la puntuación de la Asociación de Cafés Especiales de América. Utilizamos la prueba de Tukey al 5\% y correlación de Spearman para determinar el grado de asociación entre la cantidad de cafeína y la calidad sensorial. La variedad Catimor obtuvo mayor cantidad promedio de cafeína (1,214 g/100 g) proveniente del nivel altitudinal de entre 1000 - 1200 m.s.n.m, mientras que las variedades Catimor y Pache reportaron valores promedios menores de cafeína $(1,096 \mathrm{~g} / 100 \mathrm{~g})$ en muestras obtenidas entre 500 - 800 m.s.n.m. La altura tiene influencia directa positiva en el contenido de cafeína: a mayor altitud, mayor contenido de cafeína. Existe relación positiva media $(0,488)$, entre contenido promedio de cafeína y calidad sensorial de las tres variedades durante la catación del café en taza.

Citar como: Navarro Ramírez, E., PezoGonzáles, M., \& GarcíaParedes, M. (2021). Cuantificación de cafeína y su efecto en la calidad sensorial en tres variedades de café (Coffea arabica L.), según nivel altitudinal en la Región San

Martín. Revista

Agrotecnológica

Amazónica, 1(2), 4-14. https://doi.org/10.51252/ra a.v1i2.164

Recibido: $18 / 04 / 2021$

Aceptado: 18/06/2021

Publicado: 19/07/2021
Palabras clave: Cafeína, calidad sensorial, nivel altitudinal, región San Martín

Abstract. The quality of the coffee cup depends on the sensory attributes of the ground roasted bean, the amount of caffeine and the agricultural area of origin. We evaluated the effect of the altitudinal levels on the caffeine content in ground roasted coffee between the Catimor, Caturra and Pache varieties in the San Martín region and the relationship of the caffeine content on the sensory quality of coffee in the cup. Coffee samples were collected at three altitudinal levels. For the sensory analysis, the score of the Association of Specialty Coffee of America was applied. We used the $5 \%$ Tukey test and Spearman's correlation to determine the degree of association between the amount of caffeine and sensory quality. The Catimor variety obtained the highest average amount of caffeine $(1,214 \mathrm{~g} /$ $100 \mathrm{~g}$ ) from the altitude level between 1000 - 1200 m.a.s.l, while the Catimor and Pache varieties reported lower average values of caffeine $(1,096 \mathrm{~g} / 100 \mathrm{~g})$ in samples obtained. between 500 - 800 m.a.s.l. Height has a direct positive influence on caffeine content: the higher the altitude, the higher the caffeine content. There is an average positive relationship (0.488) between the average caffeine content and sensory quality of the three varieties during cupping of coffee.

Keywords: Altitudinal level, caffeine, San Martín region, sensory quality 


\section{Introducción}

La caficultura es una de las principales actividades agrícolas de la región San Martín, posesionándose como el cultivo alternativo más importante frente a la coca y el principal sustento económico de las familias, debido a que el proceso de elaboración del café se desarrolla en condiciones agroecológicas ideales. Las plantaciones se encuentran localizadas en las montañas tropicales entre los 600 y 1250 m.s.n.m. Las zonas de mayor producción son las provincias de Moyobamba, Rioja, Lamas y Tocache, donde se comercializa el grano del café pergamino en diferentes calidades, desde el tipo convencional hasta la mejor calidad de orgánico. Las variedades más difundidas son arábicas: Catimor, Caturra y Pache (Estrella Gamonal, 2015).

Frecuentemente a los consumidores les presentan un café descrito como "mezcla para el desayuno" o "mezcla gourmet". La referencia a atributos específicos de calidad es genérica e indica que el café tiene un aroma o un sabor suave. Aunque en ocasiones las mezclas efectivamente se utilizan para optimizar las características organolépticas de la bebida final por parte de especialistas, con el fin de obtener y destacar ciertos atributos o notas de sabores específicos, infortunadamente es frecuente encontrar que muchos de los productos que se venden con descriptivos de mezcla buscan reducir el costo del producto final mezclando cafés menos costosos y evitando declarar el verdadero origen del producto (Peréz Gutiérrez, 2014).

Las condiciones edafoclimáticas y de altitud, así como la variedad, un adecuado manejo agronómico y el proceso de beneficio de café influirán de manera sustanciosa en la calidad del producto, ya que sus componentes químicos resaltarán en el aroma, sabor y cuerpo del mismo. La composición química del grano del café depende de la especie, la variedad, el estado de desarrollo del fruto y el ambiente o condiciones de producción, entre otros factores. Las dos especies (Arábica y Robusta) tienen diferencias importantes en los contenidos de cafeína, trigonelia, lípidos, ácidos clorogénicos, oligosacáridos y polisacáridos, la mismas guardan relación con las características organolépticas de la bebida (FNCC, 2010).

Las limitantes para desarrollar este estudio fueron la carencia de equipos para el tostado y molienda del procesamiento de las muestras de café, además de un laboratorio de control de calidad con catadores especializados en la Universidad Nacional de San Martín; sin embargo, esto fue solucionado ya que dichos procedimientos y desarrollo de metodologías se han realizado en las instalaciones de la Cooperativa Agraria Cafetalera Oro Verde Ltda. en la ciudad de Lamas.

Esta investigación tuvo como objetivos determinar la cantidad de cafeína en café tostado molido de las variedades Catimor, Caturra y Pache en la Región San Martín, así como evaluar el efecto 
de los niveles altitudinales sobre el contenido de cafeína entre las tres variedades, además de evaluar el efecto que tiene esta cantidad de cafeína sobre la calidad sensorial del producto.

\section{Materiales y Métodos}

\subsection{Población y muestra}

Se tomaron muestras de café pergamino seco en las zonas de Moyobamba, Rioja, Lamas y Tocache, diferenciándolos por variedad y nivel altitudinal (m.s.n.m.) de procedencia. Cada muestra fue de $1000 \mathrm{~g}$, las cuales fueron tostadas y molidas. Posteriormente se efectuaron los análisis sensoriales para determinar la calidad. Estas muestras fueron recolectadas durante cuatro meses. Las cantidades de muestras por niveles altitudinales y zonas geográficas analizadas, se presentan a continuación en la tabla 1:

\begin{tabular}{|c|c|c|c|}
\hline $\begin{array}{l}\text { Altitud } \\
\text { m.s.n.m. }\end{array}$ & $\begin{array}{c}\text { Zonas cafeta- } \\
\text { leras }\end{array}$ & Variedades & $\begin{array}{c}\mathrm{N}^{\circ} \text { de } \\
\text { muestras }\end{array}$ \\
\hline \multirow[t]{3}{*}{$500-800$} & Tocache & Catimor & 05 \\
\hline & Lamas & Caturra & 05 \\
\hline & & Pache & 05 \\
\hline \multirow[t]{3}{*}{$800-1000$} & Moyobamba & Catimor & 05 \\
\hline & Rioja & Caturra & 05 \\
\hline & Lamas & Pache & 05 \\
\hline \multirow[t]{3}{*}{$1000-1200$} & Moyobamba & Catimor & 05 \\
\hline & Rioja & Caturra & 05 \\
\hline & & Pache & 05 \\
\hline Total & & & 45 \\
\hline
\end{tabular}

\subsection{Técnicas e instrumentos de recolección de datos}

Las muestras de café pergamino seco fueron recolectadas de los mismos productores, transportadas y codificadas en bolsas con cierre ziploc al laboratorio de control de calidad de la Cooperativa Agraria Cafetalera Oro Verde Ltda. para su tostado, molienda y luego realizar el análisis sensorial y determinar la cantidad de cafeína y calidad de cada muestra.

\subsection{Técnicas de procesamiento y análisis de datos}

Se utilizó un Diseño Completamente al Azar (DCA), con arreglo bifactorial de 3x3, donde el primer factor fue la variedad (Caturra, Pache y Catimor) y el segundo factor la altitud (500 a 800; 800 a 1000 y 1000 a 1200 m.s.n.m), con cinco repeticiones y con una unidad experimental de 1000 gramos de café tostado y molido.

Para el análisis estadístico de los resultados se utilizaron los paquetes estadísticos SAS versión 9.2 y Statistica Pro Ver. 13.5. Los datos fueron analizados mediante Análisis de Varianza (ANVA) y sometidos a la prueba de Tukey $(\mathrm{p} \geq 0.05)$ para determinar la naturaleza de las 
diferencias entre tratamientos. En cuanto a la contrastación de las hipótesis se utilizó la correlación rho de Spearman.

\subsection{Métodos}

Cuantificación de cafeína. Las determinaciones del contenido de cafeína en las 45 muestras de café tostado molido fueron realizadas en el laboratorio de ensayo acreditado Sociedad de Asesoramiento Técnico SAC (Lima); el cual utilizó la metodología de la AOAC 950.40 20th. Ed. (2016) - Caffeine in Roasted Coffee; conocido como Bailey - Andrew Method.

Preparación de las muestreas para catación. Para poder determinar la calidad organoléptica del café mediante el análisis sensorial, el café oro fue sometido, seleccionado y clasificado al proceso de tostado y molienda.

Tostado. Los granos de café seleccionados para la evaluación sensorial fueron tostados un día antes de la catación, se utilizaron 200 gramos de café oro por muestra, sin defectos, se tostaron únicamente los granos retenidos en los tamices entre el № 14 y 18 . El tostado se inició a una temperatura de $185^{\circ} \mathrm{C}$ llegando al final del tueste a $200{ }^{\circ} \mathrm{C}$, y el tiempo fue aproximadamente entre 10 a 12 minutos. Al finalizar el proceso, los granos fueron vertidos sobre una placa perforada para proceder al enfriado; los granos de café estuvieron a temperatura ambiente para ingresar a la molienda.

Molienda. Los granos de café fueron sometidos al proceso de molienda. Se hizo este proceso para cada muestra de café, utilizando aproximadamente $150 \mathrm{~g}$ para cada caso, ya que se necesitaba $50 \mathrm{~g}$ (10 g por cada taza) de café tostado molido para la catación y el resto fue enviado al laboratorio para la cuantificación de cafeína. Debe indicarse que entre cada una de las muestras se aseó el molino para evitar contaminaciones cruzadas.

Preparación de la bebida. Se prepararon 5 tazas de bebida por cada muestra, para ello se utilizaron $10 \mathrm{~g}$ de café tostado molido por $100 \mathrm{ml}$ de agua hirviente.

Análisis sensorial del café. Se realizó por un panel de catadores de cinco miembros, entrenados en el laboratorio de Control de Calidad de la Cooperativa Agraria Cafetalera y de Servicios Oro Verde Ltda. en Lamas, el protocolo de catación aplicado fue una adaptación de la metodología desarrollada por la Asociación Americana de Café Especial (SCAN, 2015).

Se evaluaron 10 aspectos en un rango de 6 a 10 puntos que fueron: fragancia/aroma, sabor, sabor residual, acidez, cuerpo, balance, uniformidad, taza limpia, dulzura e impresión general. La calificación de cada uno de los atributos se realizó de acuerdo a la puntuación de la Asociación de Cafés Especiales de América (SCAA, 2005) considerando como: bueno (6,00$6,75)$, muy bueno $(7,00-7,75)$, excelente $(8,00-8,75)$ y extraordinario $(9,00-9,75)$. 
Proceso de catación. En esta etapa de análisis sensorial, los catadores evaluaron únicamente las tazas con códigos, para evitar confusión en cuanto a la calificación dada a los atributos de cada muestra. La catación fue realizada para las 45 muestras de café tostado molido provenientes de las provincias de Lamas, Moyobamba, Rioja y Tocache, diferenciados por variedad y niveles de pisos altitudinales de siembra y cosecha.

El método de comparación permitió evaluar la consistencia o similitud entre las muestras. A cada una de las tazas se agregó 10 gr de café tostado molido; y se calificó la fragancia, posterior a ello se agregó agua hervida a una temperatura aproximadamente de $94{ }^{\circ} \mathrm{C}$ en forma rápida y en chorros permitiendo humedecer todos los granos molidos. Se esperó por un lapso de 4 a 5 minutos y se realizó la limpieza de taza, que consistió en quitar con cucharas la espuma que se forma en la superficie de la bebida, determinando así el aroma del café.

A los atributos fragancia y aroma se realizó una sola calificación, y se determinó la presencia de los descriptores de ambos (chocolate, dulce, floral, frutas, cítrico, melocotón, suave, maderoso, herboso y neutro). Luego, se esperó por cinco minutos hasta concebir una temperatura aproximada de $70{ }^{\circ} \mathrm{C}$ para que se sedimentaran las partículas en suspensión y así calificar las siguientes características sensoriales (acidez, sabor, cuerpo, sabor residual, balance, uniformidad, taza limpia y dulzura).

\section{Resultados y discusiones}

\subsection{Cuantificación de cafeína en diferentes variedades de café según nivel altitudinal de procedencia y su comparación estadística con la prueba de Tukey al $5 \%$}

Tabla 2

Cantidad de cafeína ( $\mathrm{g} / 100 \mathrm{~g})$ en tres variedades de café en la región de San Martín

\begin{tabular}{lcllllrr}
\hline $\begin{array}{c}\text { Zonas cafe- } \\
\text { taleras }\end{array}$ & $\begin{array}{c}\text { Altitud } \\
\text { m.s.n.m. }\end{array}$ & Variedad & $\mathbf{n}$ & $\mathbf{X}$ & DS & $\begin{array}{c}\text { Valor } \\
\text { mínimo }\end{array}$ & $\begin{array}{c}\text { Valor } \\
\text { máximo }\end{array}$ \\
\hline Tocache & $500-800$ & Catimor & 05 & 1,072 & 0.019235 & 1,05 & 0,10 \\
Lamas & & Caturra & 05 & 1,096 & 0.011402 & 1,08 & 1,11 \\
& & Pache & 05 & 1,096 & 0.011402 & 1,08 & 1,11 \\
Moyobamba & $800-1000$ & Catimor & 05 & 1,130 & 0.029154 & 1,09 & 1,17 \\
Rioja & & Caturra & 05 & 1,196 & 0.015166 & 1,18 & 1,22 \\
Lamas & Pache & 05 & 1,174 & 0.023022 & 1,15 & 1,21 \\
Moyobamba & \multirow{2}{*}{$1000-1200$} & Catimor & 05 & 1,118 & 0.028636 & 1,54 & 2,87 \\
Rioja & & Caturra & 05 & 1,214 & 0,273300 & 0,87 & 1,48 \\
& & Pache & 05 & 1.164 & 0,322630 & 0,73 & 1,42 \\
\hline n: número de muestras; X: media; SD: desviación estándar & & & &
\end{tabular}

Los datos de la tabla 2 fueron sometidos a la comparación de medias (ANOVA) mediante la Prueba de Tukey al $5 \%$, con la finalidad de establecer la existencia o no de diferencia significativa entre las mismas. 
En la figura 1 se presenta el consolidado de las cantidades de cafeínas promedios de las tres variedades por niveles altitudinales de siembra de cafetos (1: $500-800$ m.s.n.m 2: $800-1000$ m.s.n.m 3: $1000-1200$ m.s.n.m).

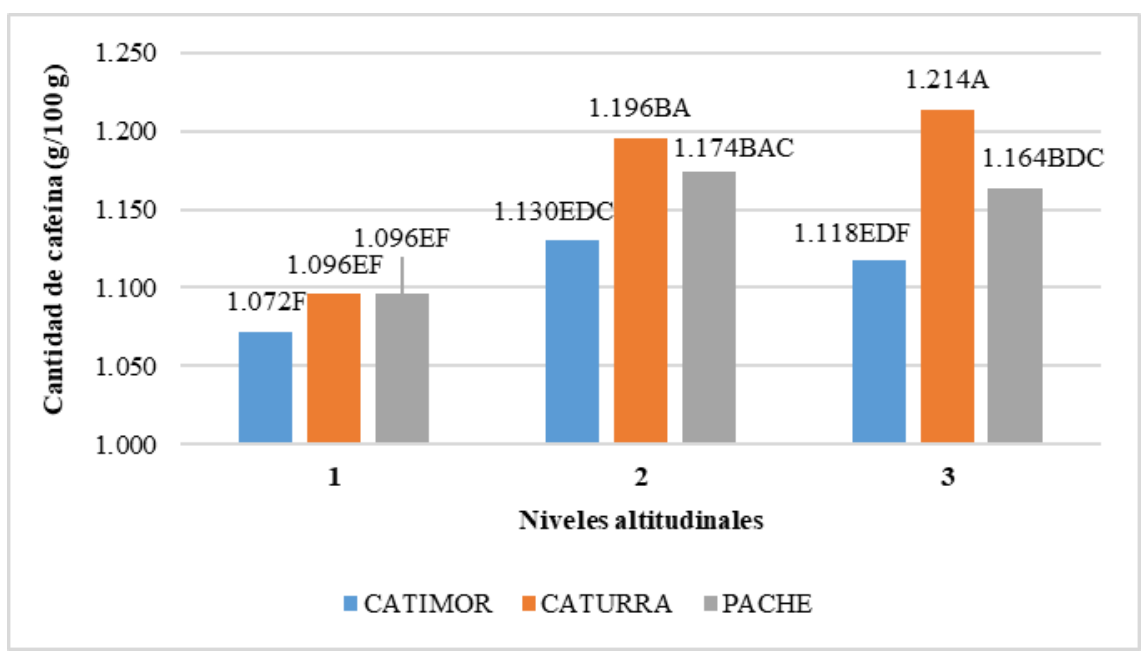

Nota: Medias con la misma letra no presentan diferencia significativa $(\mathrm{P}<0.05)$

Figura 1: Contenido de cafeína en tres variedades de café a diferentes niveles altitudinales.

Los valores del contenido promedio de cafeína de la figura 1, sometidas al análisis de varianza, obtuvieron un coeficiente de determinación (R2) de 84,31 \% y un coeficiente de variación de 1,965817. La prueba de Tukey indica que existen diferencias significativas en el contenido promedio de cafeína entre las muestras de café tostado molido de la variedad Catimor en los tres niveles altitudinales. También la variedad Caturra muestra diferencia significativa entre las muestras de los diferentes niveles altitudinales, así como el mismo análisis se realiza para la variedad Pache.

Se puede observar además que la variedad Caturra posee los niveles mayores en cuanto al contenido de cafeína, seguido de la variedad Pache, siendo la variedad Catimor la que contienen menor valor de cafeína en todos los niveles altitudinales estudiados. Esto podemos corroborar con lo expuesto por (Peréz Gutiérrez, 2014) quienes sostienen que el nivel de cafeína varía de acuerdo con la especie. Por ejemplo, el café Robusta presenta un contenido promedio de cafeína en grano seco de 2,20 \%, el café Arábica 1,20\%, la variedad Catimor 1,05\%, el Caturra 1,15\% y el Pache 1,12\%. Asimismo, podemos afirmar que en el caso de la variedad Caturra, ésta aumenta el contenido de cafeína con el nivel altitudinal de siembra de los cafetos. Esta relación también se aprecia, pero en menor proporción en las variedades Catimor y Pache.

\subsection{Evaluación sensorial de diferentes variedades de café según el nivel altitudinal de procedencia y su comparación estadística con la prueba de Tukey al $5 \%$}

En la tabla 3 se detalla los resultados promedios de la evaluación sensorial de las muestras de café en taza en las tres variedades del estudio, diferenciándolos por nivel altitudinal de 
procedencia. La evaluación sensorial nos permite encontrar y valorar todas las características que definen la calidad del café, esta bebida es el resultado de una serie de procesos por los que pasa el grano, y tiene ciertas características derivadas de un complejo número de componentes químicos (SCAN, 2015).

Tabla 3

Valores promedios de los atributos sensoriales en tres variedades de café por niveles altitudinales

\begin{tabular}{|c|c|c|c|c|c|c|c|c|c|c|c|c|}
\hline \multirow[b]{2}{*}{$\begin{array}{c}\text { Altitud } \\
\text { m.s.n.m. }\end{array}$} & \multirow[b]{2}{*}{$\begin{array}{l}\text { Variedad } \\
\text { de café }\end{array}$} & \multicolumn{10}{|c|}{ Características sensoriales } & \multirow[b]{2}{*}{ 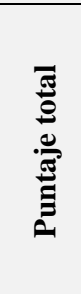 } \\
\hline & & $\underset{\tilde{z}}{\tilde{z}}$ & 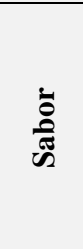 & 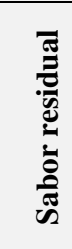 & 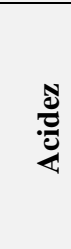 & 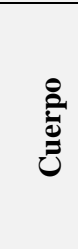 & 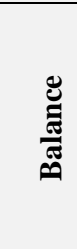 & 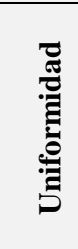 & & 晃 & 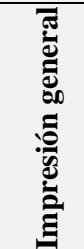 & \\
\hline \multirow[t]{6}{*}{$500-800$} & Catimor & 7,20 & 7,30 & 7,40 & 7,15 & 7,25 & 7,60 & 9,80 & 10,0 & 9,80 & 7,30 & 80,80 \\
\hline & & B & $\mathrm{CD}$ & $\mathrm{BA}$ & $\mathrm{CD}$ & $\mathrm{B}$ & A & $\mathrm{A}$ & A & A & DC & $\mathrm{D}$ \\
\hline & Caturra & 7,40 & 7,40 & 7,55 & 7,35 & 7,50 & 7,60 & 10,0 & 10,0 & 10,0 & 7,35 & 82,15 \\
\hline & & B & $\mathrm{CB}$ & $\mathrm{BA}$ & $\mathrm{CB}$ & $\mathrm{BA}$ & A & A & A & A & BDC & $\mathrm{CB}$ \\
\hline & Pache & 7,10 & 7,10 & 7,35 & 7,05 & 7,25 & 7,40 & 9,20 & 10,0 & 9,0 & 7,15 & 78,6 \\
\hline & & B & D & B & $\mathrm{D}$ & B & BA & B & A & B & D & E \\
\hline \multirow{6}{*}{$800-1000$} & Catimor & 7,35 & 7,50 & 7,50 & 7,40 & 7,45 & 7,35 & 10,0 & 10,0 & 10,0 & 7,50 & 82,05 \\
\hline & & B & $\mathrm{CB}$ & $\mathrm{BA}$ & CB & $\mathrm{BA}$ & $\mathrm{BA}$ & A & A & A & BAC & $\mathrm{CB}$ \\
\hline & Caturra & 7,45 & 7,60 & 7,60 & 7,55 & 7,60 & 7,50 & 10,0 & 9,8 & 10,0 & 7,60 & 82,7 \\
\hline & & B & B & BA & B & A & A & A & A & A & BA & B \\
\hline & Pache & 7,20 & 7,30 & 7,40 & 7,20 & 7,25 & 7,35 & 10,0 & 10,0 & 10,0 & 7,35 & 81,05 \\
\hline & & B & $\mathrm{CD}$ & BA & $\mathrm{CD}$ & B & BA & A & A & A & BDC & $\mathrm{CD}$ \\
\hline \multirow[t]{6}{*}{$1000-1200$} & Catimor & 7,50 & 7,45 & 7,50 & 7,55 & 7,40 & 7,45 & 10,0 & 10,0 & 9,80 & 7,35 & 82,0 \\
\hline & & B & CB & BA & B & BA & A & A & A & A & BDC & $\mathrm{CB}$ \\
\hline & Caturra & 8,40 & 7,90 & 7,70 & 8,00 & 7,60 & 7,40 & 10,0 & 10,0 & 10,0 & 7,65 & 84,65 \\
\hline & & A & A & A & A & A & BA & A & A & A & A & A \\
\hline & Pache & 7,30 & 7,30 & 7,40 & 7,40 & 7,45 & 7,10 & 10,0 & 10,0 & 10,0 & 7,30 & 81,25 \\
\hline & & $\mathrm{B}$ & $\mathrm{CD}$ & BA & $\mathrm{CB}$ & BA & $\mathrm{B}$ & A & A & A & DC & $\mathrm{CD}$ \\
\hline
\end{tabular}

Se observa que el único atributo sensorial similar en todas las variedades estudiadas (Catimor, Caturra y Pache) y en todos los niveles altitudinales es la taza limpia (no se presentaron diferencias estadísticas significativas). En este atributo solo la variedad Caturra de 800 - 1000 m.s.n.m presentó 9,8 de puntuación, las demás muestras obtuvieron 10 puntos, es decir mostraron transparencia de taza como ausencia de defectos y contaminaciones, o presencia de sabores o aromas ajenos la que llevan una descalificación en una taza.

En la Tabla 4, las puntuaciones promedio finales de cafés provenientes de zonas agrícolas entre 800 - 1000 m.s.n.m (Moyobamba, Rioja y Lamas) presentaron diferencias significativas entre las variedades Catimor, Caturra y Pache con puntuaciones de 82,05; 82,70 y 81,05 respectivamente, logrando obtener calificación de muy bueno en la especialidad según la escala de la SCAA. Estos resultados son similares a los obtenidos por Estrella Gamonal (2015), donde la variedad Caturra obtuvo mayor puntuación seguida de la variedad Catimor en calidad en taza en muestras procedentes de niveles altitudinales entre 800 - 1000 m.s.n.m en la zona de Lamas. 
Tabla 4

Calificación obtenida en la evaluación sensorial según la SCAA

\begin{tabular}{clrl}
\hline $\begin{array}{c}\text { Altitud } \\
\text { m.s.n.m. }\end{array}$ & $\begin{array}{c}\text { Variedad } \\
\text { de café }\end{array}$ & $\begin{array}{c}\text { Puntaje } \\
\text { total }\end{array}$ & \multicolumn{1}{c}{ Calificación } \\
\hline $500-800$ & Catimor & 80,80 & Muy bueno \\
& Caturra & 82,15 & Muy bueno \\
& Pache & 78,60 & Por debajo de la calidad \\
$800-1000$ & Catimor & 82,05 & Muy bueno \\
& Caturra & 82,70 & Muy bueno \\
& Pache & 81,05 & Muy bueno \\
$1000-1200$ & Catimor & 82,00 & Muy bueno \\
& Caturra & 84,65 & Muy bueno \\
& Pache & 81,25 & Muy bueno \\
\hline
\end{tabular}

En lo referente a las muestras de café provenientes de las zonas de 1000 - 1200 m.s.n.m (Moyobamba y Rioja), al someter al análisis de varianza y la prueba de Tukey, las variedades Catimor y Pache presentaron diferencias significativas en cuanto a la puntuación final, mientras que la variedad Caturra versus las otras dos variedades presentó diferencia altamente significativa con una puntuación promedio mayor de 84,65. En todos los casos la calificación fue de muy bueno en la especialidad de acuerdo a la escala de la SCAA.

Vaast et al. (2003) mencionaron que el café cultivado a mayor altitud suele desarrollar más atributos positivos, tales como acidez y aroma, definiendo así un mejor sabor y calidad de bebida. El grano de altura, o como lo llaman los compradores estrictamente duro, es de sabor más agradable, más parejo en conformación con un porcentaje mayor de cafés de primeras y al tostarse pierde menos peso (Duicela \& Sotomayor, 2004).

En la Tabla 5, al analizar los valores de $\mathrm{R}$ de Spearman en cuanto a los atributos de aroma, sabor, acidez y cuerpo, éstas ejercen una correlación positiva (directa) fuerte sobre el puntaje total y una correlación positiva (directa) moderada sobre la impresión general, sin embargo, la impresión general tiene relación directa fuerte con el puntaje final en la catación de cafés.

Tabla 5

Correlaciones de Spearman entre cantidad de cafeína y las variedad sensoriales

\begin{tabular}{lrcr}
\hline \multirow{2}{*}{ Variables } & Cafeína & Impresión general & Puntaje total \\
\cline { 2 - 4 } & \multicolumn{3}{c}{ Coeficientes rho (R) } \\
\hline Cafeína & 1,000000 & 0,537171 & 0,488448 \\
Aroma & 0,456053 & 0,520416 & 0,799406 \\
Sabor & 0,607024 & 0,694574 & 0,868004 \\
Sabor residual & 0,325486 & 0,518275 & 0,672974 \\
Acidez & 0,559269 & 0,598420 & 0,802715 \\
Cuerpo & 0,377393 & 0,557951 & 0,757235 \\
Balance & $-0,330873$ & 0,052122 & 0,125226 \\
Uniformidad & 0,384787 & 0,440698 & 0,546612 \\
Taza limpia & $-0,192001$ & -0.107199 & $-0,110711$ \\
Dulzura & 0,345484 & 0,489850 & 0,587726 \\
Impresión general & 0,537171 & 1,000000 & 0,785331 \\
Puntaje total & 0,488448 & 0,785331 & 1,000000 \\
\hline
\end{tabular}


El atributo taza limpia con valores de rho $-0,107199$ y $-0,110711$ frente a impresión general y puntaje total respectivamente, adquiere una correlación moderadamente débil inversa. Ahora, si analizamos la impresión general frente al puntaje total, estos tienen $\mathrm{R}$ de 0,785531 , lo cual indica que existe una relación positiva (directa) fuerte entre estos parámetros, es decir, el puntaje total incrementa proporcionalmente al mejorar la impresión general del café en taza.

El contenido de cafeína frente a los atributos de aroma, sabor, acidez, impresión general y además puntaje final tuvieron valores de $\mathrm{R}$ entre 0,4 y 0,6 , demostrando la existencia de una relación directa (positiva) moderada, es decir que, a mayor contenido de cafeína presente en las muestras de café tostado molido, se mejoraba moderadamente estos atributos señalados y por ende el puntaje total se incrementaba.

En otro análisis se observa también que el contenido de cafeína frente a los atributos sensoriales de sabor residual, cuerpo, uniformidad y dulzura, presentan valores de $\mathrm{R}$ entre 0,32 y 0,38 , lo cual estable una relación positiva (directa) baja o moderada, es decir que la cantidad de cafeína presentes en las muestras de café en taza tuvieron poca influencia o incidencia en la valoración positiva en estas características sensoriales mencionadas.

\section{Conclusiones}

Se determinaron la cantidad de cafeína en café tostado molido de las variedades Catimor, Caturra y Pache en la región San Martín, siendo la variedad Catimor la que obtuvo la mayor cantidad promedio de este alcaloide $(1,214 \mathrm{~g} / 100 \mathrm{~g})$ proveniente del nivel altitudinal de entre 1000 - 1200 m.s.n.m, mientras que las variedades Catimor y Pache reportaron valores promedios menores de cafeína $(1,096 \mathrm{~g} / 100 \mathrm{~g})$ en muestras obtenidas entre 500 - 800 m.s.n.m de nivel altitudinal de las zonas agrícolas.

El nivel altitudinal de procedencia de cafés de las variedades Catimor, Caturra y Pache en la región San Martín tuvieron influencia directa positiva en el contenido de cafeína en las mismas, lo cual indica que a mayor altitud se obtuvieron muestras con mayor contenido de cafeína en todas las variedades estudiadas con existencia de diferencias significativas entre los valores promedios encontrados.

Los resultados del coeficiente de correlación $r$ de Spearman con $p$-valor $<0,05$ entre el contenido promedio de cafeína y la calidad sensorial del café en taza de las variedades Catimor, Caturra y Pache, resultaron ser positivas del tipo media o moderada $(0,488)$, es decir que la cantidad de cafeína tiene efecto directo sobre los atributos sensoriales durante la catación del café en taza.

La variedad Caturra obtenida de zonas agrícolas entre 1000 - 1200 m.s.n.m, fue la que presentó mejores atributos sensoriales con puntuación promedio de 84,65 ; mientras que la variedad 
Pache obtenida en zonas agrícolas entre 500 - 800 m.s.n.m calificó con la menor puntuación promedio de 78,60 en calidad de café en taza.

Las variedades de café (Catimor, Caturra y Pache) en general procedentes de los tres niveles altitudinales fueron calificados como muy buenos según la escala de la SCAA y clasificados como comerciales, con grado para intercambiar en el mercado internacional, taza limpia y calidad media.

\section{Agradecimientos}

Los autores agradecen a la Universidad Nacional de San Martín - Tarapoto por el financiamiento del presente estudio a través del Instituto de Investigación y Desarrollo (IIyD) mediante el concurso de proyectos de investigación docentes 2017.

Además, al Laboratorio de Control de Calidad la Cooperativa Agraria Cafetalera y de Servicios Oro Verde Ltda. en Lamas, lugar en el cual se realizaron las pruebas de catación para determinar la calidad sensorial de las muestras de café.

\section{Referencias bibliográficas}

Duicela, I., \& Sotomayor, I. (2004). La Calidad del Café. Manual del Cultivo del Café. INIAP, FUNDRAGO, GTZ. Quevedo. Ecuador.

Estrella Gamonal, L. (2015). Evaluación física y sensorial de cuatro variedades de café (coffe arabica L.) Tolerantes a roya (Hemileia vastatrix), en relación a dos pisos eclógicos de la provincias de lamas y rioja [Universidad Nacional de San Martín - Tarapoto]. http://hdl.handle.net/11458/518

FNCC. (2010). Café de Colombia. Federación Nacional de Cafeteros de Colombia. https://www.cafedecolombia.com/particulares/manejo-agronomico/

Peréz Gutiérrez, L. B. (2014). Determinación de la calidad de tres variedades de café (Caturra, Bourbón y Typica o Criollo) de la especie c. arabica l. del municipio de Jitotol, Chiapas. [Universidad Nacional Agraria Antonio Narro]. http://repositorio.uaaan.mx:8080/xmlui/handle/123456789/533

SCAA. (2005). Protocolo para Catar. Asociación de Cafés Especiales de América.

SCAN. (2015). Evaluación sensorial del café. Plataforma Nacional de Café Sostenible Guatemala. https://xdoc.mx/preview/evaluacion-sensorial-del-cafe-5ea1fba0ac530 Vaast, P., Perriot, J., \& Cilas, C. (2003). Mejoramiento y Fortalecimiento en los Procesos de Certificación de Calidades y Comercialización del Café. Reporte. CIRAD-UNICAFE. 


\section{Conflicto de intereses}

Los autores declaramos que no existen conflictos de interés.

\section{Contribuciones de los autores}

Navarro-Ramírez, Enrique: Coordinación del proyecto, preparación de las muestras y análisis estadísticos e interpretación.

Pezo-Gonzáles, Mario: Toma de muestras en campo, coordinaciones con la catación de las muestras.

García-Paredes, María: Redacción y parte metodológica. 